$\overline{\text { Original }}$

\title{
Calorimetric Analysis of Antimicrobial Effect of p-Hydroxybenzoic Acid Alkyl Esters
}

\author{
FUMIHIRO OKADA ${ }^{\prime}$, AKI KOBAYASHI ', NOBUKI FUJIWARA', \\ NAOKO ARIMOTO ${ }^{2}$ AND KATSUTADA TAKAHASHI ${ }^{2 *}$ \\ 'Mandom Corp., Central Research Laboratories, 5-12, Juniken-cho, Chuo-ku, Osaka, \\ 540-8530 and ${ }^{2}$ Laboratory of Biophysical Chemistry, College of Agriculture, \\ Osaka Prefecture University, Sakai, Osaka 599-8531, Japan
}

Received 21 September 1998/Accepted 20 January 1999

\begin{abstract}
Calorimetric studies were conducted on the antimicrobial action of $p$-hydroxybenzoate alkyl esters (parabens), which are commomly used as preservatives in cosmetic products, and the parameters to quantitatively predict their actions on the microbial strains were determined. The result obtained was that their action on both Saccharomyces cerevisiae and Klebsiella pneumoniae became stronger as the number of carbon atoms in the alkyl chain increased. When comparing the affinity of the drugs to both microorganisms based on the drug concentration required to inhibit growth activity by $50 \%$, a linear relationship was observed to exist between the number of carbon atoms in an alkyl chain and the affinity. In addition, the same drug exhibited a higher effect on S. cerevisiae than on $K$. pneumoniae, which may be due to the difference in the membrane structures of the microorganisms.
\end{abstract}

Key words: Preservative/Microbial calorimetry/Antimicrobial effect/Specific growth activity/Apparent binding affinity/Cosmetics and toiletries

\section{INTRODUCTION}

Like in food products, preservatives are added to in cosmetic products to prevent microbial contamination (Moral, 1992; Richardson, 1981; Tanaka, 1997). Moreover, antibacterial agents are contained in quasidrugs and medical drugs in addition to preservatives with the aim of disinfecting the skin, and eliminating specific bacteria such as those causing acne, dandruff, and body odor (Charig et al., 1991; Haustein et al., 1993; Korting et al., 1991).

In using such preservatives and antibacterial agents in cosmetic products and quasi' or medical drugs, it is important to choose the most suitable kind of agent according to the characteristics of the product and purposes of its use (Corbett, 1992, 1997). However, actions of preservatives and antibacterial agents are not completely understood, and, at present, the selection of agents to be used is based

${ }^{*}$ Corresponding author. Tel and Fax : +81-722-50-0525 largely upon past experience. In recent medical drug development, knowledge of the relationship between the molecular design of drugs (drug design) and antimicrobial actions has become extremely important in the development of drugs which are effective against bacteria causing nosocomial infections (methicillin-resistant Staphylococcus aureus, MRSA, and vancomycin-resistant Enterococcus, VRE) (Hashimoto, 1996; Sasatsu et al., 1996; Takeda et al., 1996).

In order to help resolve of these problems, we investigated a method for qualitatively analyzing actions of antimicrobial drugs by microbial calorimetry. Microbial calorimetry is a method for monitoring the growth of microorganisms by determining the metabolic heat generated as a result of microbial growth from a non-destructive point of view (Antoce et al., 1996a; Takahashi, 1996). Since this microbial growth thermogram $[g(t)$ curve] accurately reflects the growth of microorganisms, changes in the patterns of growth thermograms obtained from the culture system to which a drug has been added can provide 
various kinds of information about the drug effects. Therefore, analyses of these changes by an appropriate method can provide a quantitative understanding of the drug effects which conventional methods cannot (Antoche et al., 1996b, 1997a, 1997b, 1998; Koumoto et al., 1996; Okuda et al., 1996). Accordingly, we investigated the antimicrobial actions of $p$ hydroxybenzoate alkyl esters (parabens) by microbial calorimetry. $p$-Hydroxybenzoate alkyl esters are widely used as preservatives in food and cosmetic products as well as in quasi- and medical drugs, have a broad antibacterial spectrum, and are considered to be very safe (Hatanaka, 1976; Matsuda et al., 1993; Richardson, 1981). To determine growth-inhibiting parameters for the actions of parabens, we obtained growth thermograms using a multiplex batch calorimeter (Takahashi, 1996) having 24 calorimetric units, in order to investigate the growth behavior of Klebsiella pneumoniae and Saccharomyces cerevisiae in the growth culture containing various concentrations of parabens. We then calculated the 50\% growth inhibition concentration $\left(K_{\theta}\right)$ on the basis of retardation in growth, using growth thermograms of $K$. pneumoniae and $S$. cerevisiae obtained from each drug sample. Then, we drew a drug potency curve according to the drug concentration dependency, and evaluated the drug effects.

Moreover, we determined the affinity of each drug to $K$. pneumoniae and $S$. cerevisiae microbial cells (changes in Gibbs free energy of binding) on the basis of the $50 \%$ growth inhibition concentration $\left(K_{\theta}\right)$, and examined the morphology of molecules and drug effects. Methyl, ethyl, propyl, butyl, pentyl, and hexyl $p$-hydroxy benzoate were used as parabens.

\section{MATERIALS AND METHODS}

\section{Devices and samples}

For determining the metabolic heat of a microorganism, we used a multiplex batch calorimeter containing 24 calorimetric units (Bio Thermo Analyzer H-201, Nippon Medical \& Chemical Instruments Co., Ltd., Osaka; abbreviated as BTA) which was previously reported by Takahashi (1996).

Six commercially available antibacterial $p$-hydroxybenzoate alkyl esters (parabens) were used. The microorganisms employed in the study were our stock strain of yeast, $S$. cerevisiae no.9302, and $K$. pneumoniae which had been isolated from a cosmetic material. S. cerevisiae was incubated in a glucose peptone medium (Nippon Seiyaku, pH5.6-5.8) and K. pneumoniae was incubated in a brain heart infusion medium (NISSUI, $\mathrm{pH}$ 7.2).

\section{Procedure}

In the experiment, $5 \mathrm{ml}$ of the glucose peptone medium or the brain heart infusion medium each containing a paraben at a specified concentration was placed in a $30 \mathrm{ml}$ incubation vial which served also as a calorimetric vessel. $50 \mu \mathrm{l}$ of a cell suspension at $10^{3}$ cells $/ \mathrm{ml}$ was added and stirred, and each vial was placed in a calorimetric unit kept at $30^{\circ} \mathrm{C}$ to determine the heat evolution signals which result from growth.

\section{RESULTS AND DISCUSSION}

Figure 1( I) shows the growth thermogram of the yeast, $S$. cerevisiae, incubated for $48 \mathrm{~h}$ in the medium containing propyl paraben at a concentration of 0 to $0.0168 \%(\mathrm{w} / \mathrm{v})$. This thermogram will to be referred to as the $g(t)$ curve. The ordinate represents the thermal electromotive force $(\mu \mathrm{V})$ determined by a thermal detector, and the abscissa represents the incubation time $(h)$. The $g(t)$ curve shown in this figure exhibits changes in the pattern of the growth thermogram associated with changes in the propyl paraben concentration. As the propyl paraben concentration increases, the incubation time required for
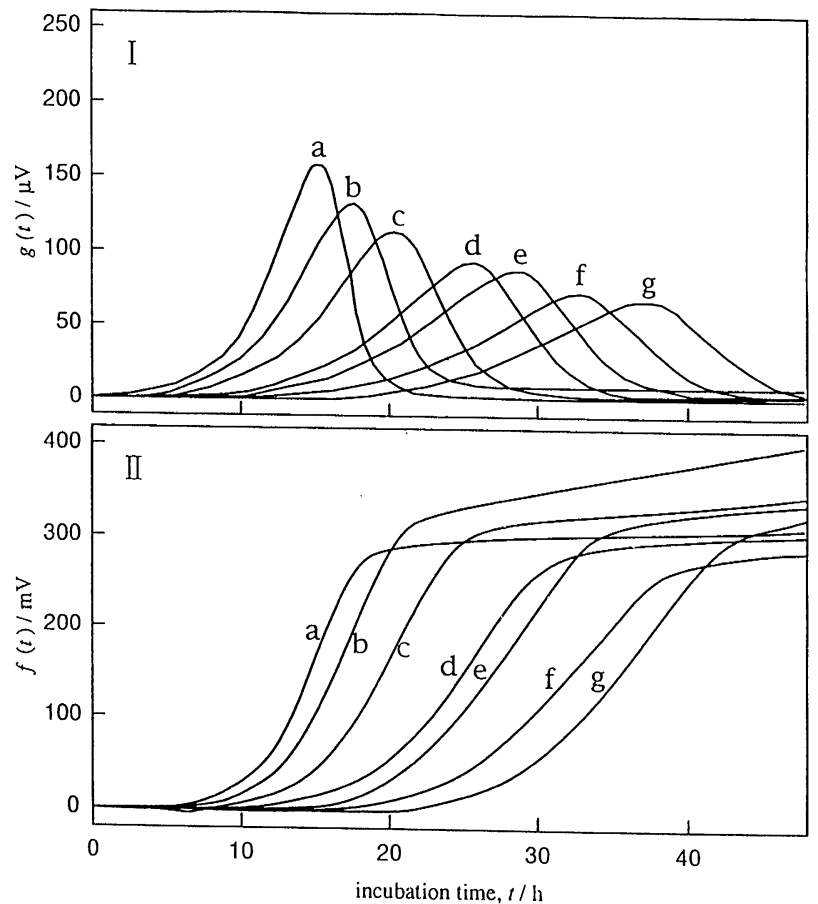

FIG. 1. Growth thermograms (I) and growth curves (II) observed for the growing culture of Saccharomyces cerevisiae in brain-heart infusion medium containing various amounts of propylparabens. The 7 curves shown in each panel correspond from left to right to the concentration of propylparaben. a, 0; b, 0.0072; c, 0.0102; d, 0.0132; e, $0.0144 ; f, 0.0156 ; g, 0.0168 \%(w / v)$. 
the peak of the microbial growth thermogram to be reached becomes longer, and the inclination at the beginning of the curve becomes smaller, suggesting inhibition of the microbial growth.

The $g(t)$ curve shows the heat evolution process associated with the microbial growth in the calorimetric unit. This, however, shows only apparent changes in heat, and the loss of the heat to the surroundings is also included in the results. When the heat conduction constant (Newton's cooling constant) of the calorimetric unit including the sample is denoted by $K$, the true amount of heat generated $[f(t)]$, corrected for the heat loss arising from heat exchange with the surroundings, can be obtained by the following equation (Takahashi, 1996).

$$
f(t)=g(t)+K \int g(t) \mathrm{d} t \quad(1)
$$

In other words, the $g(t)$ curve obtained here can be converted into a calorimetrically obtained growth curve using a simple algorithm (Antoce et al., 1996, 1997a; Takahashi, 1996). The curve thus obtained is shown in Fig. 1 (II) as the $f(t)$ curve. As is clear from this figure, the initial phase of the growth includes a portion which shows exponential growth, and this can be applied to the following exponential growth equation to obtain the growth rate constant $\mu$ (Antoce et al., 1996a; Hashimoto et al.,1982; Takahashi, 1996).

$$
f(t)=A N_{0} e^{\mu t}+B N_{0}
$$

In this equation, $N_{0}$ denotes the initial cell count, and $A$ and $B$ are constants. When the growth rate constant $\mu$ is used, the specific growth activity $\left(\mu_{\mathrm{i}} / \mu_{\mathrm{m}}\right)$ can be obtained from the ratio of the growth rate constant, $\mu_{i}$, at the drug concentration, $i$, to the growth rate constant, $\mu_{\mathrm{m}}$, at the drug concentration, 0 . Thus, a drug potency curve can be drawn from the equation (3), and the drug potency characteristics can be quantitatively described (Antoce et al., 1996a, 1997a, 1997b).

$$
\mu_{\mathrm{i}} / \mu_{\mathrm{m}}=1 /\left\{1+\left(i / K_{\mu}\right) m^{\mu}\right\}
$$

In this equation, $K_{\mu}$ denotes the mean value of the dissociation constant of the drug per site. While $m_{\mu}$ can be regarded as the number of sites, it is also an exponent which expresses the synergism of drug actions. Accordingly, $\mu_{i}$ is given by a hyperbolic function as the $m_{\mu}$-th power of the drug concentration, i. (Antoce et al.,1996b).

As shown in Figs. 1 ( I ) and 1 (II), the drug effects are expressed as changes in the patterns of the $g(t)$ and $f(t)$ curves, which imply the above-mentioned changes in the growth rate constant, and retardation in the growth.

While the $f(t)$ curve indicates time courses of heat evolution during the microbial growth, the differential value, $f^{\prime}(t)$, is considered to express the growth activity as measured from the metabolic heat at each instant of incubation time based on the caloric generation. Here we define that the incubation time required for the microbial activity, $f^{\prime}(t)$, to reach a certain value $\alpha$, in the absence of the drug is expressed as $t_{\alpha}(0)$, and that the incubation time required for the same activity level to be reached at the drug concentration $i$, is expressed as $t_{\alpha}(i)$. Then, the specific growth activity based on retardation in the growth is expressed by the ratio $t_{\alpha}(0) / t_{\alpha}(i)$ (Antoce et al., 1996a, 1996b, 1997a, 1997b, 1998a). The specific growth activity based on retardation in the growth thus defined can be treated in the same way as the microbial activity based on the growth rate constant. The following equation, which is similar to equation (3), can be written for $t_{\alpha}(0) / t_{\alpha}(i)$,

$$
t_{\alpha}(0) / t_{\alpha}(i)=1 /\left\{1+\left(i / K_{\theta}\right)^{m_{\theta}}\right\}
$$

where the parameters $m_{\mu}$ and $K_{\mu}$ were renamed as $m_{\theta}$ and $K_{\theta}$, respectively (Antoce et al.,1996a).

Equations (3) and (4) suggested that both the specific growth activity based on the growth rate constant $\left(\mu_{i} / \mu_{m}\right)$, and the specific growth activity based on retardation in growth $\left(t_{\alpha}(0) / t_{\alpha}(i)\right)$ can be mathematically given by the same function pattern, and that the drug potency curve can be drawn not only from $\mu_{i} /$ $\mu_{\mathrm{m}}$, but also from $t_{\alpha}(0) / t_{\alpha}(i)$ using equation (4) (Antoce et al., 1996a; 1996b). However, it should be noted that the specific growth activity based on the growth rate constant does not completely match with the specific growth activity based on retardation in growth. For example, if a drug works bacteriostatically, then only the retardation in growth will be observed while the growth rate constant $\mu$ would be unaffected (Okada et al, 1998). Thus the discrepancy between the two parameters $\mu_{i} / \mu_{\mathrm{m}}$ and $t_{\alpha}(0) / t_{\alpha}(i)$ reflects essential differences related to the mechanism of action of the dug (parabens in the case of this study), as well as errors associated with optimization. In reality, all kinds of drugs exert bacteriocidal actions at concentrations higher than a certain level, and often completely inhibit the growth of microorganisms. Furthermore, if we look at the antimicrobial action through the growth rate constant, then the effect of bactericidal actions will be disregarded. In other words, $t_{\alpha}(0) / t_{\alpha}(i)$ appears to be more appropriate in the case of drugs having both bacteriostatic and bacteicidal actions. Therefore, we used the specific growth activity based on retardation in growth, $t_{\alpha}(0) /$ $t_{\alpha}(i)$, in discussing and comparing antimicrobial actions of parabens in this article.

Figure 2( I ) shows the potency curves for six parabens as measured from retardation in growth of $S$. cerevisiae, including the experimental data of propyl paraben obtained from Figs. 1( I ) and (II). The ordinate in Fig. 2 represents the specific growth 
activity based on retardation in growth, $t_{\alpha}(0) / t_{\alpha}(i)$, and the abscissa represents the logarithm of the drug concentration. Plots represent experimental data and solid lines represent regression curves, i.e., drug potency curves. As is evident from this figure, the drug potency curves for parabens represented as $t_{\alpha}(0) /$ $t_{\alpha}(i)$ shows the shift to the left in the order of methyl, ethyl, propyl, butyl, pentyl and then hexyl. This indicates that the antibacterial activity is the highest for methyl, followed by ethyl, propyl, butyl, pentyl and then hexyl. Figure 2(II) shows the drug potency curves as measured from retardation in growth of $K$. pneumoniae. Similarly to the results with $S$. cerevisiae, the stronger antibacterial effects were observed when the alkyl chain was longer, but changes in drug potency curves were not as marked as those for $S$. cerevisiae. These findings suggest that, although the antibacterial effects become stronger when the alkyl chain is longer, the increase in the antibacterial effects is not as significant as that observed with $S$. cerevisiae. The drug potency curves are shown only for methyl, ethyl, and propyl parabens in this figure, because the data of drugs having an alkyl chain longer than butyl could not be obtained due to limitations in the solubility (Hatanaka, 1976). The results obtained with S. cerevisiae and K. pneumoniae have revealed that changes in the pattern of the growth thermogram become more evident if drug potency curves are drawn.

When drug potency curves are drawn, the drug concentration inducing 50\% growth inhibition $\left(K_{\theta}\right)$ should be determined simultaneously (Antoce et al., 1996a, 1997a, 1997b; Takahashi, 1996). $K_{\theta}$ is an inhibition parameter representing the effective drug

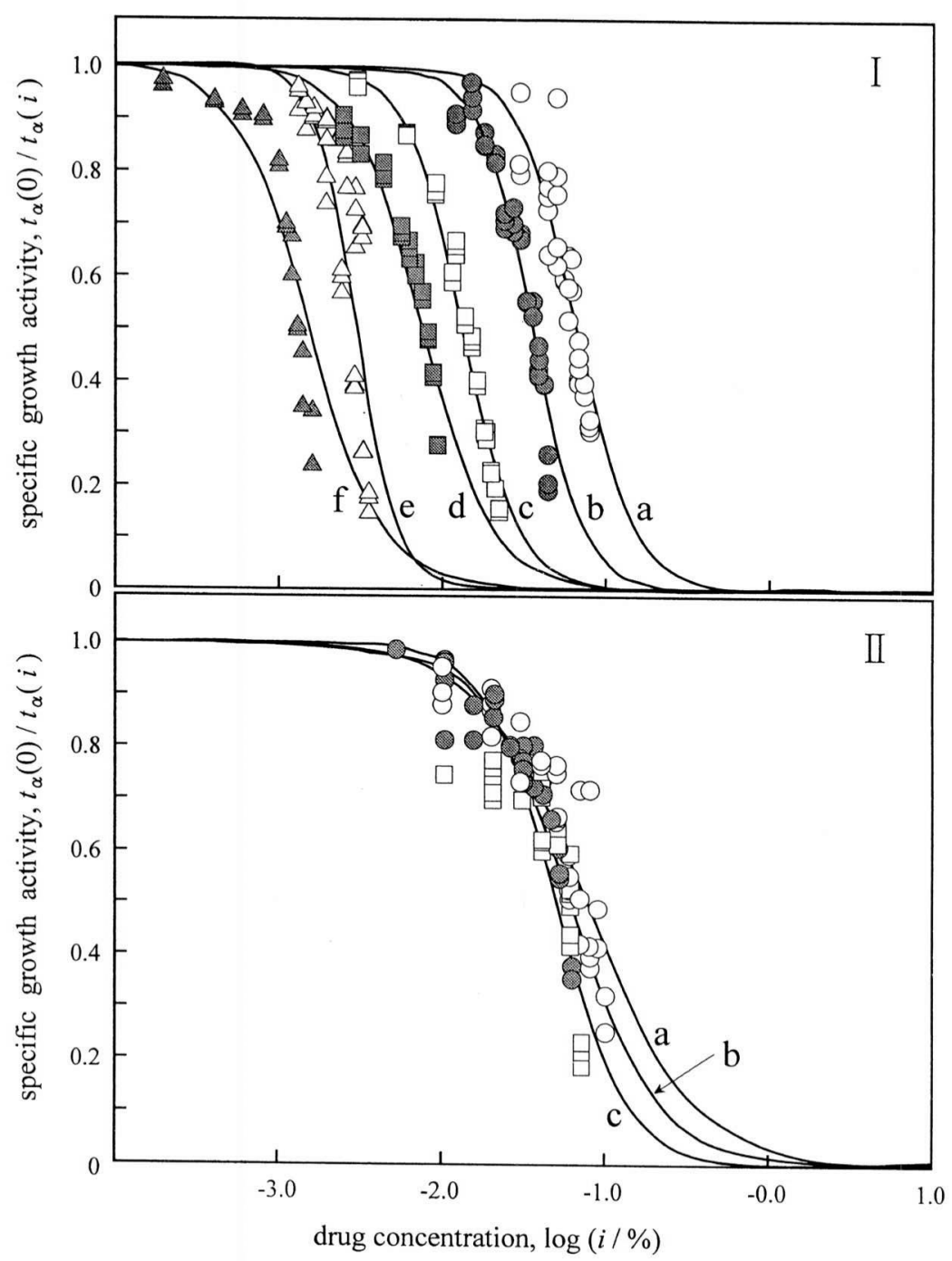

FIG. 2. The specific growth activity as a function of drug concentration. Drugs are p-hydroxybenzoate alkyl ester. a, methl ester; b,ethyl ester; c, propyl ester; d, butyl ester; e, pentyl ester; f, hexyl ester. ( I ) Saccharomyces cerevisiae, (II) Klebsiella pneumoniae. 
concentration at which the growth activity is reduced by half, and is equivalent to the median concentration of the drug potency curve. The values of $K_{\theta}$ thus obtained are summarized in the fourth column of Table 1.

In addition to the parameter $K_{\theta}$, it is also possible to obtain the minimum inhibition concentration from the drug concentration dependency of the growth rate (Antoce et al., 1996a). If we define that the reduction of the growth activity by a drug is expressed as (1 $\left.t_{\alpha}(0) / t_{\alpha}(i)\right)$, and that it is regarded as being in proportion to the $m_{\mathrm{i}}$-th power of the drug concentration, $i$, we can obtain the following equation

$$
1-t_{\alpha}(0) / t_{\alpha}(i)=k_{i} i^{\mathrm{mi}}
$$

where, $k_{i}$ is a proportional constant.

According to the equation (5), the drug concentration at which the growth activity is completely lost can be obtained by the following equation (Antoce et al., 1996a, 1997a, 1997b; Takahashi, 1996).

$$
i_{\mathrm{c}}=\left(1 / k_{\mathrm{i}}\right)^{1 / \mathrm{mi}}
$$

The two unknown values $\left(k_{i}\right.$ and $\left.m_{i}\right)$ can be determined by the regression analysis on equation (5) and $i_{c}$ can be calculated by equation (6). Needless to say, the value of $i_{c}$ can be regarded as the minimum inhibition concentration by the nature of the problem. In the fifth column of Table 1 are given the values of MIC determined by the above procedure. In order to compare the antimicrobial activity of parabens more quantitatively, their apparent affinity to microbial cells were determined.

On the basis of the above-mentioned $50 \%$ growth inhibitory concentration, $K_{\theta}$, the apparent affinity of parabens to microbial cells $(A)$, or in other words, the changes in the Gibbs free energy of binding, was obtained using the Gibbs equation (Nitta et al., 1971; Nitta et al., 1996)

$$
A=R T \ln K_{\theta} \quad(7)
$$

where $R$ is the gas constant and $T$ is the absolute tem- perature. The values obtained are shown in the last column of Table 1. It is obvious that the apparent affinity increases with increasing numbers of alkyl carbons in both actions against both $S$. cerevisiae and $K$. pneumoniae.

Figure 3 shows plots of the drug affinity to the two microorganisms obtained. As is evident from the figure, an extremely favorable linear relationship is noted between the number of carbons in the alkyl chain and the drugs affinity. Moreover, differences in the antibacterial potency appears to be mainly attributable to the carbon chain. The increase in the inhibitory actions of parabens against microbial cells in association with increases in the number of carbons in the alkyl chain seems to strongly imply that hydrophobic interactions with cells are highly responsible for this.

Moreover, from the slope of the plots, the degree of affinity of drugs to cells was estimated to be $2.1 \mathrm{~kJ} /$ mol per carbon in the case of $S$. cereviciae, and $1.1 \mathrm{~kJ}$ $/ \mathrm{mol}$ in the case of $K$. pneumoniae. It was shown by various investigators in literature that among microbes, there is a large variety in membrane structures and their composition (Kabara, 1984). The discrepancy obtained above seems to be most reasonably explained by the differences in the membrane structures of $S$. cerevisiae and $K$. pneumoniae, and it may be assumed that the higher inhitory effect on a microorganism cell observed with a paraben having a larger number of carbon atoms in its alkyl chain is attributable substantially to its hydrophobic interaction with the cell.

We have discussed the qualitative analysis of the potency of antimicrobial drugs in this study, citing parabens, which are commonly used as preservatives in foods, cosmetic products and medical drugs, as an example. It is clear that highly reliable information can be provided by the present method based on the measurement of microbial metabolic heat. As is rep-

TABLE 1 . Fifty $\%$ growth inhibitory concentration $\left(K_{\theta} / \mathrm{mM}\right)$, minimum inhibitory concentration $\left(\mathrm{MIC}_{\theta} / \mathrm{mM}\right)$ and apparent binding affinities $\left(A / \mathrm{kJ} \mathrm{mol}^{-1}\right)$.

\begin{tabular}{clrrr}
\hline $\begin{array}{l}p \text {-Hydroxybenzoate } \\
\text { alkyl ester }\end{array}$ & \multicolumn{1}{c}{ Strain } & $K_{\theta} / \mathrm{mM}$ & $\mathrm{MIC}_{\theta} / \mathrm{mM}$ & $A / \mathrm{kJ} \mathrm{mol}^{-1}$ \\
\hline \multicolumn{1}{c}{ Sodium salt } & S. cerevisiae & 112.98 & 206.21 & 5.5 \\
& K. pneumoniae & 69.70 & 126.15 & 6.7 \\
Methyl & S. cerevisiae & 4.47 & 6.69 & 13.6 \\
& K. pneumoniae & 6.85 & 18.48 & 12.6 \\
Ethyl & S. cerevisiae & 2.26 & 3.34 & 15.4 \\
& K. pneumoniae & 5.02 & 9.80 & 13.4 \\
Propyl & S. cerevisiae & 0.88 & 1.29 & 17.7 \\
& K. pneumoniae & 2.77 & 4.28 & 14.9 \\
Butyl & S. cerevisiae & 0.39 & 0.66 & 20.0 \\
Pentyl & S. cerevisiae & 0.20 & 0.29 & 21.5 \\
Hexyl & S. cerevisiae & 0.07 & 0.11 & 24.2 \\
\hline
\end{tabular}



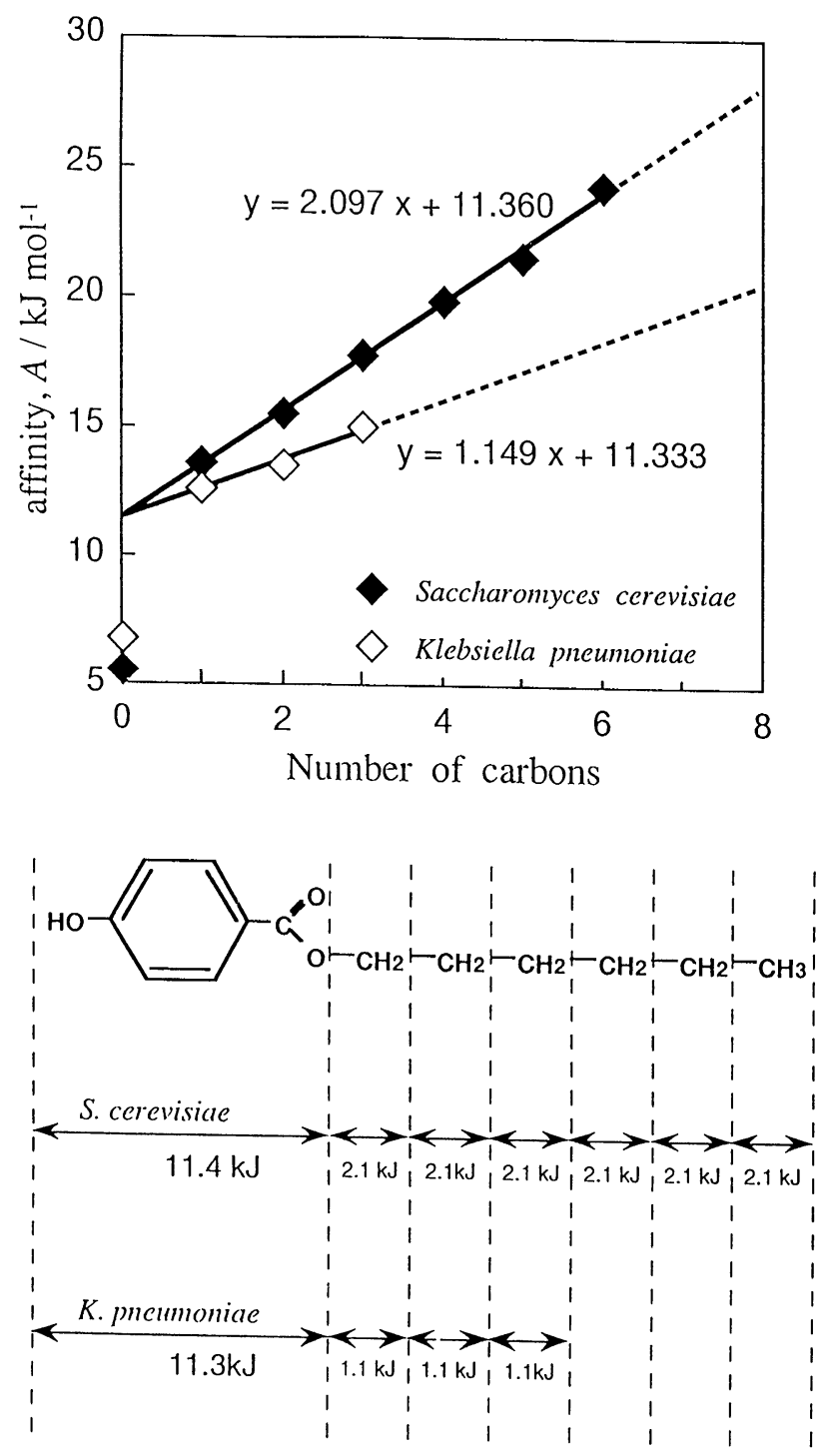

FIG. 3. Relationship between the number of alkyl carbons in $p$ - hydroxybenzoate alkyl ester and the apparent drug affinity to the microbial cell.

resented by Fig. 3, examination of the details about structure-activity relationship is also possible. In light of the results presented here, we believe that this technique is effective not only for accurate evaluation of drug potency, but also for new approaches of molecular design (Okada et al, 1996b, 1998) and even the evaluation of synergistic effect of drugs (Antoce et al, 1998b). We also believe that the usefulness of analyses of antimicrobial actions by the calorimetric method in development of medicines will be recognized even more strongly in the future and that the methodology described here will contibute to introduce a new insight into the study of antimicrobial drugs.

\section{REFERENCES}

Antoce, O. -A., Pomohaci, N., Antoce, V., Fukada, H., Takahashi, K., Kawasaki, H., Amano, N., and Amachi, T. (1996a) Application of calorimetry to the study of ethanol tolerance of some yeast strains. Biocontrol Sci., 1, 3-10.

Antoce, O. -A., Antoce, V., Takahashi, K.,Nitta, Y., Fukada, H., and Kawasaki, H. (1996b) Quantitative analysis of the action of ethanol on growth activity of yeast and its theoretical background. Netsu Sokutei, 23, 45-52.

Antoce, O. -A., Antoce, V., Takahashi, K., and Yoshizako, F. (1997a) Quantitative study of yeast growth in the presence of added ethanol and methanol using a calorimetric approach. Biosci. Biotechnol. Biochem., 61, 664-669.

Antoce, O. -A., Antoce, V., Takahashi, K., Pomohaci, N., and Namolosanu, I. (1997b) Calorimetric determination of the inhibitory effect of $\mathrm{C}_{1}-\mathrm{C}_{4} n$-alcohols on growth of some yeast species. Thermochim Acta, 297, 33-42.

Antoce, O. -A., Antoce, V., Pomohaci, N., Namolosanu, I. and Takahashi, K. (1998a) Inhibitory effect of decanoic acid on yeast growth at various $\mathrm{pHs}$ and ethanol concentrations. Biocontrol Sci.,3,7-15.

Antoce, O. -A., Antoce, V., Mori, N., Yasui, S., Kobayashi, A., and Takahashi, K. (1998b) Calorimetric evaluation of the antimicrobial properties of 1,3butanediol and 1,2-pentanediol on various microorganisms. Netsu Sokutei, 25, 2-8.

Charig, A., Froebe, C., Simone, A. and Eigen, E. (1991) Inhibitor of odor-producing axillary bacterial exoenzymes, J. Soc. Cosmet. Chem., 42, 133-145.

Corbett, R. J.(1992) Preservation of cosmetics and toiletries: A microbiological overview. Parfum. Kosmet., 73, 22-27.

Corbett, R. J.(1997) Formulation characteristics and preservative efficacy. SOFW J., 123, 246-258.

Hashimoto, H. (1996) Studies on Staphyrococcus aureus: from the past to the future (in Japanese). Nippon Saikingaku Zasshi, 51, 841-852.

Hashimoto, M., and Takahashi, K. (1982) Calorimetric studies of microbial growth: Quantitative relation between growth thermograms and inoculum size. Agric. Biol. Chem., 46, 1559-1564.

Hatanaka, K. (1976) Antimicrobial activity and security of $p$-hydroxybenzoic acid esters and sorbic acid (in Japanese). Fragrance J., 19, 61-67.

Haustein, U. -F., Herrmann, J., Hoppe, U., Engel, W., and Sauremann, G. (1993) Growth inhibition of coryneform bacteria by a mixture of three natural products. J. Soc. Cosmet. Chem., 44, 211-220.

Kabara, J. J. (1984) Cosmetic and Drug Preservation 
Principles and Practice. Marcel Dekker, Inc.,21-27. Korting, H. -C., Greiner, K., Hubner, K., and Hamm, G. (1991) Changes in skin $\mathrm{pH}$ and resident flora by washing with synthetic detergent preparations at pH5.5 and 8.5. J. Soc. Cosmet. Chem., 42, 147158.

Koumoto, Y., Arimoto, N., Fukada, H., Nitta, Y., Fukada, H., Takahashi, K., and Kawasaki, H. (1996) Calorimetric study of the action of preservatives on the putrefaction process of boiled soybeans (in Japanese). Bokin Bobai, 24, 9-15.

Matsuda, H., Sawahara, H., and Watanabe, T. (1993) Combination effects of four $p$-hydroxybenzoic acid esters (in Japanese). Bokin Bobai, 21, 63-68.

Moral, J. (1992) Cosmetic microbiology. Cosmet. Toilet., 107, 65-72.

Nitta, Y., Mizushima, M., Hiromi, K., and Ono, S. (1971) Influence of molecular structure of substrates and analogues of Taka-amylase A catalyzed hydrolyses. I . Effect of chain length of linear substrates. J. Biochem., 69, 567-576.

Nitta, Y., Shirakawa, M., and Takahashi, Y. (1996) Kinetic study of active site structure of $\beta$-amylase from Bacillus cereus var. micoides. Biosci. Biotechnol. Biochem., 60, 823-827.

Okada, F., Kobayashi, A., Fujiwara, N., Matsuyama, K., and Takahashi, K. (1996a) Quantitative study about the action of preservatives by microbial calorimetry. Proceedings of 2nd Scientific Conference of Asian Societies of Cosmetic Scientists, Seoul, Korea, pp.626-637.

Okada, F., Kobayashi, A., Fujiwara, N., and Taka- hashi, K. (1996b) Calorimetric study on the inhibitory actions on $p$-hydroxybenzoic acid alkyl esters against microbial cells. Proceedings of 14th IUPAC Conference on Chemical Thermodynamics,pp.364.

Okada, F., Kobayashi, A., Fujiwara, N., and Takahashi, K. (1999) Bacteriostatic and bactericidal actions of antimicrobial drugs studied by microbial calorimetry, Biocontrol Sci., 4, 35-39.

Okuda, S., Takahashi, K., Fukada, H., Nitta, Y., Nakao, H., and Kirihara, M. (1996) Calorimetric study of the inhibitory effect of some boron derivatives on the growth activity of yeast (in Japanese). Bokin Bobai, 24, 649-655.

Richardson, E. L. (1981) Update-frequency of preservative use in cosmetic formulas as disclosed to FDA. Cosmet. Toilet., 96, 91-92.

Sasatsu, M. (1996) Antiseptic-Resistance in Staphy/ococcus aureus (in Japanese). Nippon Saikingaku Zasshi, 51, 755-765.

Takahashi, K. (1996) Design of a non-destructive system to quantitatively evaluate the antibacterial and antifungal action of drugs (in Japanese). Bokin Bobai, 24, 313-320.

Takeda, T., Yoshino, K., Ramamurthy, T., Uchida, H., Matsuda, E., and Pal, A. (1996) Molecular epidemilogy of infections with enterohemorrhagic Escherichia coli 0157 in Japan (in Japanese). Nippon Saikingaku Zasshi, 51, 1037-1042.

Tanaka, K.(1997) Microbial contamination control of shampoos, hair rinses and body soaps (in Japanese). Nippon Keshohin Gijutsusha Kaishi, 31, 245-253. 